\title{
PERCEPÇÃO SOBRE O APRENDIZADO DE SAÚDE COLETIVA E O SUS ENTRE ALUNOS CONCLUDENTES DE CURSO DE ODONTOLOGIA
}

\author{
PERCEPTIONS ON THE LEARNING OF COLLECTIVE HEALTH AND THE SUS AMONG STUDENTS \\ WHO WERE COMPLETING THE COLLEGE OF DENTISTRY
}

\author{
Luiz Roberto Augusto Noro ${ }^{1}$ \\ Sara Melo Torquato ${ }^{2}$
}

Resumo O objetivo deste artigo é apresentar a visão de alunos concludentes de curso de Odontologia sobre o aprendizado na área da Saúde Bucal Coletiva e conhecer sua percepção sobre o Sistema Único de Saúde (SUS). A quase totalidade dos alunos indicou a importância dos conteúdos trabalhados nas disciplinas da área da Saúde Bucal Coletiva, reconheceu como adequada a forma de abordagem feita pelos professores, apontou os aspectos positivos do SUS e vislumbrou uma perspectiva real de se inserir neste campo de trabalho. Entretanto, a grande maioria tem maior interesse nas atividades clínicas tradicionais, mesmo quando pensa em sua inserção no SUS, possivelmente estimulada pela grande quantidade de disciplinas da área clínica nos cursos de Odontologia. Para efetivação do SUS, é fundamental o envolvimento do profissional de saúde no fortalecimento do controle social, em especial na aliança com a população assim como na gestão dos serviços. Em função disto, a formação em Odontologia deve aprofundar sua discussão nestes elementos e na perspectiva da clínica ampliada, permitindo que as conquistas do SUS tragam benefícios concretos tanto para os profissionais de saúde, no desempenho de sua função de transformador da realidade social, quanto para a população, na conquista de seus direitos constitucionais.

Palavras-chave saúde pública; aprendizagem; educação em odontologia.
Abstract The purpose of this article is to present the perspective of students who were completing the college of Dentistry about their learning in the Collective Oral Health area, and to get to know their perception about the Single Health System (SUS). Almost all of the students mentioned the importance of the content that was worked on in Public Oral Health, acknowledged that the way their professors approached the issue was appropriate, noted the positive aspects of the SUS, and saw a real prospect of entering this field of work. However, most of them are more interested in traditional clinical activities, even while considering entering the SUS. This is possibly driven by the large amount of clinical subjects offered by the colleges of Dentistry. In order for the SUS to be effective, it is essential to get health professionals involved in strengthening social control, particularly in the alliance with the population and in service management. Because of this, training in Dentistry must deepen its discussion regarding those factors and about the enhanced clinic perspective, allowing the achievements made by the SUS to afford concrete benefits both to healthcare professionals, while performing their function of transforming the social reality, and to the population in the conquest of their constitutional rights.

Keywords public health; learning; education in dentistry. 


\section{Introdução}

Contemplar o sistema de saúde vigente do Brasil é uma das mais importantes competências e habilidades proposta pelas Diretrizes Curriculares Nacionais dos Cursos de Graduação em Odontologia (Brasil, 2002). Para viabilizar tal formação é essencial que o curso de Odontologia tenha professores efetivamente vinculados aos princípios do Sistema Único de Saúde (SUS), com elevado compromisso com a sociedade e capacitados a estimular os alunos para a compreensão destes elementos em sua possível futura inserção profissional.

Grande parte dos cursos de Odontologia tem situado tal discussão na área da Saúde Bucal Coletiva, cujo referencial, segundo Narvai (1992), é a atenção à saúde bucal, a qual é constituída pelo conjunto de ações que, incluindo a assistência individual, não se esgota nela, buscando atingir grupos populacionais através de ações de alcance coletivo que objetivam manter a saúde bucal. Tal perspectiva, no entender de Sanchez, Drumond e Vilaça (2008), constitui um desafio para o cirurgião-dentista que, como participante da equipe de saúde, deve levar em consideração o envolvimento de seus atos com os aspectos sociais, políticos, econômicos e culturais importantes para o processo de consolidação desta proposta de atenção à saúde, pensando-se na inserção na Estratégia Saúde da Família. Sua atuação em equipes de saúde multidisciplinares, desenvolvendo atividades de promoção e educação em saúde que contemplem simultaneamente a saúde integral de indivíduos e coletividades, na percepção de Aerts, Abegg e Cesa (2004), proporcionará uma elevação dos níveis de saúde da população.

Com a consolidação do SUS e com a expansão da Estratégia Saúde da Família, é bastante provável para Lazzarin, Nakama e Cordoni Júnior (2007) que as Instituições de Ensino Superior (IES) tenham que repensar os seus currículos para formar profissionais que atendam às necessidades socioeconômicas do país, melhorando, assim, os índices de saúde.

Com isto, o real papel da universidade, na visão de Garbin et al. (2006), seria o de identificar corretamente os problemas de saúde de cada município ou região e dizer como poder resolvê-los, devendo o ensino e a pesquisa serem direcionados para ações de impacto social que possibilitassem melhores condições de vida para a população. Assim, para os autores, os egressos estariam preparados para o mercado de trabalho e, ao mesmo tempo, cientes do seu compromisso de devolver à sociedade tudo o que lhes foi ensinado. Nesta mesma perspectiva, Silva, Vargas e Ferreira (2004) indicam que o intuito seria garantir uma escola integrada com o serviço de saúde, que garantisse educação orientada para o ser humano e que formasse um profissional qualificado do ponto de vista científico, técnico, crítico, humano e ético, atuante e comprometido socialmente com a luta pela saúde de seu povo. 
Entretanto, para Araújo e Dimenstein (2006), são raros os egressos que fazem opção pelo trabalho na Estratégia Saúde da Família, por sua filosofia e proposta de atenção à saúde, contrariando seus ideais primários de realização profissional. Concordando com esta visão, Albuquerque et al. (2005) identificam que, estimulados pelas transformações globais no mercado de trabalho, em decorrência, entre outros fatores, da queda do poder aquisitivo da população brasileira, os dentistas estão buscando de modo crescente ocupação junto ao serviço público.

Apesar disso, estudos como os de Thind, Atchison e Andersen (2005) e de Medeiros Júnior (2005) mostram a efetividade de atividades realizadas em atividades extramurais, tendo como foco a saúde bucal coletiva, os quais trazem grandes perspectivas de inovação e concretização no que se refere à integração docente-assistencial e compreensão da necessidade dos alunos em desenvolver trabalhos voltados à comunidade. Ainda para Galassi et al. (2006), o aluno em contato com comunidades carentes, além do aprendizado, exerce cidadania, tornando-se um profissional mais humano.

Considerando estas premissas, o presente artigo, que se origina de pesquisa desenvolvida durante a monitoria em Saúde Bucal Coletiva, teve como objetivo conhecer a percepção dos alunos do curso de Odontologia da Universidade de Fortaleza (Unifor) sobre o aprendizado na área da Saúde Bucal Coletiva, assim como identificar a percepção do aluno sobre o SUS.

\section{Metodologia}

A pesquisa que deu origem a este artigo caracteriza-se como observacional descritiva, do tipo transversal. A população total investigada constituiu-se de 104 alunos cursando o último ano do curso de Odontologia da Unifor. Para composição da amostra, considerando a margem de erro de $15 \%$ e efeito de desenho igual a 2, foram identificados de forma aleatória 47 alunos, sendo 21 do nono semestre (Estágio Extramural I) e 26 do décimo semestre (estágio Extramural II).

Para a coleta dos dados foi desenvolvido um questionário semiestruturado aplicado sob a forma de entrevista. Todas as entrevistas foram aplicadas por uma única pesquisadora, o que permitiu uma homogeneidade na forma de questionar os alunos, trazendo validade para os dados coletados.

Anteriormente à aplicação do questionário, a pesquisadora discutiu exaustivamente com o coordenador da pesquisa os critérios e códigos utilizados, buscando evitar-se viés da coleta de dados. Para atestar a adequação do instrumento e realizar os ajustes necessários, foi realizado estudo-piloto com dez alunos, sendo cinco de cada semestre, antes do início da coleta de dados.

As variáveis independentes presentes no estudo foram o sexo e a faixa etária. As variáveis de interesse explanatório disseram respeito ao aprendizado em 
relação às disciplinas da Saúde Bucal Coletiva, as características dos professores desta área de conhecimento, particularidades da disciplina, importância da Saúde Bucal Coletiva para a prática profissional, a percepção do aluno sobre o SUS e sua possível atuação na Estratégia Saúde da Família.

Os dados foram submetidos à análise bivariada da distribuição das situações previstas nas variáveis expressas em porcentagem e à análise estatística ( $\mathrm{p}<0,05)$, sendo o banco de dados da pesquisa construído na plataforma SPSS versão 13.0 .

Visando a garantir proteção aos participantes da pesquisa, uma vez que o a investigação foi realizada com seres humanos, o projeto de pesquisa foi encaminhado ao Comitê de Ética em Pesquisa da Universidade de Fortaleza, tendo sido aprovado.

\section{Resultados}

Do total de alunos pesquisados, $36(76,6 \%)$ eram do sexo feminino e 11 $(23,4 \%)$ do sexo masculino, em idades que variavam de 21 a 29 anos, sendo que $22(46,8 \%)$ na faixa etária de 21 a 23 anos, 24 (51,0\%) na de 24 a 29 anos e um $(2,2 \%)$ sem informação relativa à idade.

Quanto ao aprendizado nas disciplinas, 43 dos entrevistados (91,5\%) identificaram-no como satisfatório ou muito satisfatório. Ao mesmo tempo, na avaliação do conjunto dos professores das disciplinas de Saúde Bucal Coletiva, apenas um aluno $(2,1 \%)$ considerou a abordagem desses professores inadequada. No questionamento relativo às particularidades das disciplinas, observou-se que $14,9 \%$ (sete alunos) não se recordavam da aula mais interessante que tiveram na área de Saúde Bucal Coletiva, enquanto 55,3\% (26 alunos) a identificaram em atividades do Estágio Extramural, ou seja, próximo da conclusão do curso de graduação. A identificação da importância desta área de conhecimento para a prática profissional foi praticamente universal entre os respondentes, uma vez que 95,7\% (45 alunos) consideraram-na muito importante.

A percepção dos alunos em relação ao SUS foi positiva para 80,9\% dos participantes, assim como $85,1 \%$ veem sua inserção nesse sistema como provável ou muito provável, sendo a atividade clínica a perspectiva desta inserção para $61,7 \%$ desses alunos. Outro elemento importante foi em relação à possível forma de inserção na Estratégia Saúde da Família, na qual, daqueles que responderam, apenas seis alunos (13,3\%) pretendem se envolver exclusivamente com o gerenciamento, enquanto a grande maioria $(64,4 \%)$ entende que sua inserção dar-se-ia em atividades ligadas essencialmente à clínica. Parte dos alunos (22,3\%) admite que poderia se inserir na clínica ou no gerenciamento. Nenhuma destas variáveis apresentou dife- 
rença estatisticamente significante em relação ao sexo ou à faixa etária, o que pode ser observado na Tabela 1. Vale ressaltar que os dados apresentados na tabela a seguir referem-se apenas aos alunos que responderam às questões propostas.

Tabela 1

Distribuição das variáveis sobre atuação no SUS, de acordo com faixa etária e sexo, Universidade de Fortaleza, 2008

\begin{tabular}{|c|c|c|c|c|c|c|c|c|c|c|}
\hline \multirow{3}{*}{ Atuação no SUS } & \multicolumn{4}{|c|}{ Faixa etária } & \multicolumn{6}{|c|}{ Sexo } \\
\hline & \multicolumn{2}{|c|}{ 21-23 anos } & \multicolumn{2}{|c|}{ 24-29 anos } & \multirow[b]{2}{*}{$\mathrm{p}$} & \multicolumn{2}{|c|}{ Masculino } & \multicolumn{2}{|c|}{ Feminino } & \multirow[b]{2}{*}{$p$} \\
\hline & $\mathrm{n}$ & $\%$ & $\mathrm{n}$ & $\%$ & & $\mathrm{n}$ & $\%$ & $\mathrm{n}$ & $\%$ & \\
\hline \multicolumn{11}{|l|}{ Inserção ESF } \\
\hline Provável & 19 & 41,3 & 20 & 43,5 & 1,000 & 10 & 21,3 & 20 & 63,8 & 0,894 \\
\hline Improvável & 3 & 6,5 & 4 & 8,7 & & 1 & 2,1 & 6 & 12,8 & \\
\hline \multicolumn{11}{|l|}{ Percepção ESF } \\
\hline Positiva & 17 & 37,0 & 20 & 43,5 & 0,884 & 9 & 19,1 & 29 & 61,7 & 1,000 \\
\hline Negativa & 5 & 10,9 & 4 & 8,7 & & 2 & 4,3 & 7 & 14,9 & \\
\hline \multicolumn{11}{|l|}{ Gerenciamento } \\
\hline Sim & 7 & 15,9 & 8 & 18,2 & 1,000 & 5 & 11,1 & 11 & 24,4 & 0,670 \\
\hline Não & 14 & 31,8 & 15 & 34,1 & & 6 & 13,3 & 23 & 51,1 & \\
\hline
\end{tabular}

Fonte: Os autores.

\section{Discussão}

Apesar da grande importância da Saúde Bucal Coletiva visando à formação do acadêmico de Odontologia com perspectivas para atuação no SUS, a grande maioria dos cursos de Odontologia do Brasil ainda apresenta em seus currículos uma predominância da área clínica. Tentando fazer uma interlocução destes campos de conhecimento, o currículo do curso de Odontologia da Unifor buscou, segundo Noro et al. (2005), a construção de uma proposta curricular vislumbrando o crescimento contínuo de complexidade e a aplicação na realidade social.

Outro objetivo do estudo era o de verificar a visão dos alunos em relação ao SUS e sua possível inserção como profissional de saúde nesse sistema. Foi bastante importante verificar que a grande maioria dos alunos, apesar de uma visão ainda preconceituosa de alguns atores da academia, representados por alguns professores, observa aspectos positivos do SUS. O SUS é o principal mecanismo de conquista de direitos relativos à universalização da 
atenção à saúde e deve ser defendido de forma clara por todos aqueles que acreditam em princípios tais como ética, cidadania e justiça social. Outro elemento que chamou a atenção foi a clareza da grande maioria dos alunos que vislumbra no SUS uma perspectiva real de campo de trabalho.

A oportunidade de primeiro emprego do cirurgião-dentista recém-formado na Estratégia Saúde da Família pode ser evidenciada em pesquisa de Morita, Haddad e Araújo (2010), que identificam 34\% dos profissionais destas equipes na faixa etária de 20 a 29 anos.

Segundo Chaves e Silva (2007), entretanto, a força do campo privado de origem é ainda superior, o que exige um esforço extra para a manutenção das mudanças requeridas na prática profissional para sua maior efetividade no campo público. Considerando esta realidade, é fundamental que políticas públicas sejam desenvolvidas para superar as expectativas negativas ainda apresentadas pelos futuros profissionais de saúde, permitindo sua efetiva participação na consolidação do SUS como real campo de defesa da saúde da população.

Cabe ressaltar o efeito da grande oferta de abordagem de conhecimentos da clínica nos currículos de Odontologia: apesar de a quase totalidade dos alunos classificar a abordagem da Saúde Bucal Coletiva como interessante ou muito interessante, identificar o desempenho dos professores desta área como positiva e quase todos identificarem a área como importante para sua prática profissional, a atividade clínica ainda desperta o interesse da maioria dos alunos $(61,7 \%)$. É preocupante observar que os profissionais disponíveis no mercado de trabalho, na visão de Garbin et al. (2006), são ainda preparados dentro de uma concepção que privilegia a abordagem individual e curativa e são, portanto, incapazes de transcender o espaço do consultório e propor um diagnóstico sobre o coletivo e suas intervenções, em uma abordagem integralizadora, sendo que o 'ensino odontológico flexneriano' é ainda praticado na grande maioria dos cursos de graduação do país.

Tal evidência fica clara em artigo de Araújo e Dimenstein (2006), em que perceberam que os cirurgiões-dentistas pesquisados têm perfil profissional voltado para a clínica, aspecto que está relacionado a sua formação acadêmica, mas também à própria expectativa da comunidade, bem como da gestão do serviço em priorizar o atendimento clínico.

Não se trata, entretanto, de abordar a clínica e a saúde coletiva como campos opostos. O grande desafio para os cursos de Odontologia seria o de formar profissionais de saúde com uma visão onde a clínica abordaria as doenças tanto em sua dimensão biológica quanto em uma perspectiva ampliada, na visão de Campos e Amaral (2007), identificando situações que ampliam o risco ou vulnerabilidade das pessoas, mas sempre centradas no problema de saúde em sujeitos, que vivem em comunidades. 
Cordioli e Batista (2007) salientam a falta de articulação da teoria com a prática, uma visão da Odontologia descontextualizada da realidade com consequente despreparo para atuação no mercado de trabalho, uma formação inadequada para o trabalho no contexto do SUS, um preparo inadequado para ações ligadas à administração e ao gerenciamento da própria prática e pouco preparo para o relacionamento com o paciente e com os outros profissionais da área.

Sabe-se que hoje um dos grandes desafios para efetivação do SUS é a importância do envolvimento do profissional de saúde na corresponsabilidade da gestão dos serviços e no estímulo à participação popular. Presta, Saliba e Garbin (2007) confirmam o desconhecimento por parte dos gestores a respeito da importância do planejamento para o enfrentamento das necessidades de saúde bucal da população. Da mesma forma, Kasper (2002), em pesquisa com gestores do serviço público, conclui que suas dificuldades relacionam-se com os aspectos jurídico/administrativo, ideológico/conceitual e no tipo de prática de saúde do SUS.

Por isso, além da necessidade, apontada por Albuquerque et al. (2007), da utilização da educação permanente dos profissionais de saúde na produção de políticas descentralizadoras, coerentes com os princípios do SUS, é papel dos cursos de Odontologia aprofundar a discussão sobre estes aspectos, permitindo que as conquistas do SUS possam trazer realização tanto para os profissionais de saúde, no desempenho de sua função de transformador da realidade social, quanto para a população, elemento fundamental na conquista da cidadania.

\section{Notas}

1 Professor adjunto do Departamento de Odontologia da Universidade Federal do Rio Grande do Norte (UFRN), Natal, Rio Grande do Norte, Brasil. Doutor em Ciências da Saúde pela Universidade Federal do Rio Grande do Norte. <noro@ufrnet.br> Correspondência: Departamento de Odontologia da Universidade Federal do Rio Grande do Norte, Av. Salgado Filho, 59, Natal, Rio Grande do Norte, Brasil, CEP 59056-000.

2 Graduanda do Curso de Odontologia da Universidade de Fortaleza (Unifor), Fortaleza, Ceará, Brasil. <saramtorquato@gmail.com> 


\section{Referências}

AERTS, Denise; ABEGG, Claídes; CESA, Kátia. O papel do cirurgião-dentista no Sistema Único de Saúde. Ciência \& Saúde Coletiva, Rio de Janeiro, 2004, v. 9, n.1, p. 131-138.

ALBUQUERQUE, Sandra H. Carvalho et al. Como você se sente? A experiência do Estágio Extramural do Curso de Odontologia da Unifor. In: NORO, Luiz Roberto Augusto (Org.). Curso de Odontologia da Unifor: 10 anos ensinando e aprendendo. Fortaleza: Universidade de Fortaleza, 2005.

ALBUQUERQUE, Verônica Santos et al. Integração curricular na formação superior em saúde: refletindo sobre o processo de mudança nos cursos do Unifeso. Revista Brasileira de Educação Médica, Rio de Janeiro, 2007, v. 31, n. 3, p. 296-303.

ARAÚJO, Yanne Pinheiro de; DIMENSTEIN, Magda. Estrutura e organização do trabalho do cirurgião-dentista no PSF de municípios do Rio Grande do Norte. Ciência \& Saúde Coletiva, Rio de Janeiro, 2006, v. 11, n. 1, p. 219-227.

BRASIL. Ministério da Educação. Diretrizes Curriculares Nacionais dos Cursos de Graduação em Odontologia. Resolução CNE/CES 3, de 19 de fevereiro de 2002. DOU, Brasília, DF, 4 mar. 2002. Seção 1, p. 10.

CAMPOS, Gastão Wagner de S.; AMARAL, Márcia Aparecida do. A clínica ampliada e compartilhada, a gestão democrática e redes de atenção como referenciais teórico-operacionais para a reforma do hospital. Ciência \& Saúde Coletiva, Rio de Janeiro, 2007, v. 12, n. 4 , p. $849-859$.

CHAVES, Sônia C. Lima; SILVA, Lígia M. Vieira da. As práticas profissionais no campo público de atenção à saúde bucal: o caso de dois municípios da Bahia. Ciência \& Saúde Coletiva, Rio de Janeiro, 2007, v. 12, n. 6, p. 1697-1710.

CORDIOLI, Otavio F. Genta; BATISTA, Nildo Alves. A graduação em Odontologia na visão de egressos: propostas de mudanças. Revista da Abeno, São Paulo, 2007, v. 7, n. 1, p. 88-95.

GALASSI, Marlei A. Seccani et al. Atividades extramuros como estratégia viável no processo ensino-aprendizagem. Revista da Abeno, São Paulo, 2006, v. 6, n. 1, p. 66-69.

GARBIN, Cléa A. Saliba et al. O papel das universidades na formação de profissionais na área de saúde. Revista da Abeno, São Paulo, 2006, v. 6, n. 1, p. 6-10.

KASPER, Elisabete. Gestão pública em odontologia dificuldades e perspectivas. Revista da Faculdade de Odontologia, Porto Alegre, 2002, v. 43, n. 2, p. 35-39.

LAZZARIN, Helen Cristina; NAKAMA, Luiza; CORDONI JÚNIOR; Luiz. O papel do professor na percepção dos alunos de Odontologia. Saúde e Sociedade, São Paulo, 2007, v. 16, n. 1, p. $90-101$.

MEDEIROS JÚNIOR, Antonio et al. Experiência extramural em hospital público e a promoção da saúde bucal coletiva. Revista de Saúde Pública, São Paulo, 2005, v. 39, n. 2, p. 305-310.

MORITA, Maria Celeste; HADDAD, Ana Estela; ARAÚJO, Maria Ercília. Perfil atual e tendências do cirurgião-dentista brasileiro. Maringá: Dental Press, 2010.

NARVAI, Paulo Capel. Saúde bucal: assistência ou atenção?. São Paulo: FSP-USP, 1992.

NORO, Luiz Roberto Augusto et al. Projeto pedagógico: a construção coletiva. In: (Org.). Curso de Odontologia da Unifor: 10 anos ensinando e aprendendo. Fortaleza: Universidade de Fortaleza, 2005.

PRESTA, Andréia Antoniuk; SALIBA, Orlando; GARBIN, Cléa A. Saliba. A saúde bucal nos planos municipais de saúde: análise da $8^{a}$ Regional de Saúde do estado do 
Paraná. Revista da Faculdade de Odontologia, Passo Fundo, 2007, v. 12, n. 1, p. 27-31.

SANCHEZ, Heriberto Fiúza; DRUMOND, Marisa Maia; VILAÇA, Ênio Lacerda. Adequação de recursos humanos ao PSF: percepção de formandos de dois modelos de formação acadêmica em Odontologia. Ciência \& Saúde Coletiva, Rio de Janeiro, 2008, v. 13, n. 2, p. 523-531.

SILVA, Aline Mendes; VARGAS, Andréa Maria Duarte; FERREIRA, Efigênia e Ferreira e. Integridade da atenção e o ensino superior. Arquivos do Centro de Estudos do Curso de Odontologia, Belo Horizonte, 2004, v. 40, n. 3, p. 245-256.
THIND, Amardeep; ATCHISON, Kathryn; ANDERSEN, Ronald. What determines positive student perceptions of extramural clinical rotations? An analyziz using 2003 ADEA Senior Survey Data. Journal of Dental Education, 2005, v. 69, n. 3, p. 355-62.

Recebido em 06/05/2010

Aprovado em 15/07/2010 